\title{
Quality of Nursing Care in Saudi Arabia: Are Empathy, Advocacy, and Caring Important Attributes for Nurses?
}

\author{
Abdulaziz M. Alsufyani ${ }^{1,2}$, Abdulaziz A. Aldawsari ${ }^{3}$, Sayer M. Aljuaid ${ }^{2}$, \\ Khalid E. Almalki ${ }^{4}$, Yasir M. Alsufyani ${ }^{5}$ \\ ${ }^{1}$ College of Nursing, King Saud University, Saudi Arabia \\ ${ }^{2}$ Taif Comprehensive Rehabilitation Center, Ministry of Human Resources and Social Development, \\ Riyadh, Saudi Arabia \\ ${ }^{3}$ Medical Services Administration, Ministry of Human Resources and Social Development, \\ Riyadh, Saudi Arabia \\ ${ }^{4}$ Primary Health Care Center, Ministry of Health, Riyadh, Saudi Arabia \\ ${ }^{5}$ College of Nursing, King Khaled University, Abha, Saudi Arabia \\ Corresponding Author: Abdulaziz.M. Alsufyani (a.aziz987@hotmail.com)
}

\begin{abstract}
Background: The examination of the literature indicates that practicing nurses are barely engaged in developing enhancement programs for quality nursing care. There are numerous studies on the value of nursing care, but none offers ways of assessing the value of care provided by nurses. Identifying the essence of quality nursing care can facilitate effective enhancement approaches. It was prudent to explore the relationship between advocacy, caring, and empathy in delivering quality nursing care.

Purpose: This study aimed to comprehend the views of Saudi Arabian nurses on how empathy, advocacy, and caring act as measurements of quality of nursing care.

Methods: A qualitative investigative, descriptive design was used to explore the advocacy, empathy, and caring from the viewpoints of practicing nurses. Twenty-one general and specialized medical care nurses from King Saud Medical City in Saudi Arabia were recruited through purposive sampling. The researchers conducted semistructured interviews that were recorded, written out, and subjected to thematic analysis. Results: The findings have led to the establishment of quality Saudi nursing care with the identification of core themes: empathy, advocacy, and caring. The findings of this study elevate the understanding of the quality of nursing care in the Saudi context.

Conclusion: The participants aver that patient advocacy, empathy, and care are parts of the characteristics of nursing profession. These characteristics aid in listening and comprehending patients' perspectives. Following the findings, it is suggested to provide training to the nurses to overcome the challenges faced by nurses in reflecting empathy.
\end{abstract}

Keywords: Advocacy, caring, empathy, quality nursing care, Saudi Arabia

How to Cite: Alsufyani, A. M., Aldawsari, A. A., Aljuaid, S. M., Almalki, K. E., \& Alsufyani, Y. M. (2020). Quality of nursing care in Saudi Arabia: Are empathy, advocacy, and caring important attributes for nurses?. Nurse Media Journal of Nursing, 10(3), 244-259. doi:10.14710/nmjn.v10i3.32210

Permalink/DOI: https://doi.org/10.14710/nmjn.v10i3.32210 


\section{BACKGROUND}

In the domain of clinical practice, a significant rise has been observed in the moral factors involved in quality patient care. As healthcare organizations are becoming very competitive and quality care improvement is dominant, healthcare providers and policymakers are increasingly recognizing the importance of exploring the indicators of quality care for the improvements in the healthcare processes. The central interest has been regarded in following the patient-centered approach, where patients' perspectives and preferences are highly respected (Austin, 2000).

Nurses serve as a critical part of the overall healthcare system, as they offer care to individuals, families, and communities. Nsiah, Siakwa, and Ninnoni (2019) noted that nurses could empower patients, reduce unnecessary treatment, ease patients' discomforts, and may serve as a shield against inadequate care. Evidence related to optimal nursing care indicates that advocacy, empathy, and caring are essential components of nursing practice. Ratka (2018) outlined empathy as the most important human characteristic that is valuable in every aspect of life. Patient-centered care is initiated by various empathic healthcare practitioners. A significant correlation between physicians' empathy towards patients improved patients' adherence to medication, increased satisfaction, and positive treatment outcomes have been well developed (Hannan et al., 2019). Duarte, Pinto-Gouveia and Cruz (2016) defined empathy as "the ability of an individual to consider others' feelings" (p.2).

A positive correlation between physicians' empathy and other domains of affective care has been observed (Heidke, Howie \& Ferdous, 2018). Ratka (2018) added that students that are empathetic possess stronger affective skills and are capable to develop, acquire, display and reinforce strong behaviors, attitudes, and abilities that are highly required for effective patient care. The study further suggested that the level of empathy is further effective and is generally influenced by educational interventions that are being taught during the educational process. The usefulness of the educational methods can be further enhanced through activities that help students in enhancing their affective skills, followed by the increase in empathetic skills. Kim (2018) stated that empathy provides a better clinical environment and promotes patient engagement and treatment compliance. It also helps to reduce the occurrence of medical errors and enables nurses to devise a more accurate nursing care plan based on the actual needs of their patients.

Duarte et al. (2016) conducted a study to examine the relationship between selfcompassion and empathy and professional quality of life (compassion fatigue, satisfaction, and nurses' burnout). The findings of the study outlined the influence of empathy and self-compassion on three different domains of the quality of life. A positive association between empathetic concern and compassion fatigue and compassion satisfaction was observed. These effects were observed through the negative components of self-compassion and self-kindness, and common humanity, which also acted as the significant moderators. A similar association was detected between compassion fatigue and personal distress. Through the study findings, it was suggested that increased affective empathy might serve as a risk factor for compassion fatigue. Kuipers, Cramm and Nieboer (2019), in their study, provided a strong emphasis on the usefulness of patient-centered care and morbidity and highlighted that the factors 
are important for improved healthcare outcomes. It further examined the relationship between patient-centered care, physical well-being, co-creation of care, social wellbeing, and patients' satisfaction. Findings of the study indicated a positive association between patient-centered care and co-creation of care with patients' satisfaction with care along with their social and physical well-being.

Advocacy, on the other hand, can be defined as nurses' act or way of working, which includes considerations on the best interest of their patients. It can be understood through different perspectives of providing patient support, which in many cases may be in the form of protection of patients' rights, while the protection and comfort of patients for ethical dialogues between patients. Patient advocacy has received greater attention in several dimensions within healthcare organizations. Davoodvand, Abbaszadeh, and Ahmadi (2016) stated that patient advocacy is a vital feature in the nursing practice as it relies on several factors such as human engagement, moral distress, and social relationships. For instance, Aupia, Lee, Liu, Wu, and Mills (2018) have stated that safeguarding patients against illegal and unethical acts was considered an element of patient advocacy. Likewise, Abbasinia, Ahmadi, and Kazemnejad (2020) have claimed that patient advocacy issues cannot be resolved by only supporting the patient, but should address the administrative and systematic care matters. However, it has been emphasized that nurse character traits have a bearing on the efficiency of patient advocacy. In addition, Dadzie, Aziato, and Aikins (2017) claims that nurses' advocacy might be directed towards patients perceived as vulnerable, intimated, complaining, uninformed, or disinterested in their care. Abbasinia et al. (2020) conducted a literature review to provide a clear definition of patient advocacy. Through a detailed review of literature, different attributes of patient advocacy have been identified, which includes: providing information to patients regarding their diagnosis, prognosis, treatment outcomes, and providing alternatives regarding patients' healthcare treatment, followed by the discharge program. It further includes providing value to the management of selfcontrol, maintaining humanity and individuality, providing patients the choice regarding their preferred treatment, managing individuality, patient privacy, humanity and acting on patients' beliefs, culture, values, and preferences preferring the cultural values among healthcare team members, acting as patients' voice. It also further contributes to the promotion of social justice, which includes the implementation of policies and rules while confronting the inappropriate aspects in them (Abbasinia et al., 2020).

For James and Mill (2018), caring reflects the core component of nursing profession. While there is no consensus on how to define the concept of caring, most nursing researchers tended to define "caring behaviors" instead of defining "caring". Therefore, the current study acknowledges the caring behaviors described by Salimi and Azimpour (2013) and indicated that "caring behaviors are actions concerned with the well-being of a patient, such as sensitivity, comforting, attentive listening, honesty, and nonjudgmental acceptance" (p.269). In that essence, caring behaviors are expected from the professional nurses in order to maximize the quality of the delivered care.

Considering the findings of previous studies, it is evident that empathy, advocacy, and caring behaviors are essential indicators of the quality of nursing care. In addition, nurses are aware of the contribution of quality nursing caring to the patients, the 
institution, laws, and professional standards, as well as how it improves the patients' experience (Karaca \& Durna, 2019). Although nurses' meanings of empathy, advocacy, and caring behaviors are useful to explore how the quality of nursing care is, previous studies failed to support the meaning of these indicators among nurses (Abbasinia et al., 2020; Elewa, ElAlim, \& Etway, 2016; Moudatsou, Stavropoulou, Philalithis, \& Koukouli, 2020). It is worth mentioning that if current literature on quality criteria failed to illustrate the meaning of quality nursing care for nurses, this might be contributing to the inhibits the process of improvement in the quality of nursing care. Yet, there is a gap in the literature about indicators of the quality of nursing care globally, and particularly in Saudi Arabia.

\section{PURPOSE}

This study aimed to uncover the meaning of empathy, advocacy, and caring among Saudi nurses as attributes of quality nursing care.

\section{METHODS}

\section{Study design}

This study utilized a qualitative phenomenological approach. A descriptive design was followed to discover the nursing traits that have a bearing on patient advocacy, empathy, and care from the insights of working nurses. This design focuses on gathering an explicit understanding of human behavior and causes that hold such action from the participants' feedback. It also depends on narratives from participants to address the issues.

\section{Setting}

The study was carried out at the biggest medical city in Saudi Arabia, King Saud Medical City (KSMC) in Riyadh, Saudi Arabia, that provides general and specialist medical care with the capacity of 1,400 beds. The participants in this study were selected from the Med/Surg, OB/GYN, Oncology, and Cardiology wards of the hospital.

\section{Ethical consideration}

Before the commencement of the meeting with the participants, the researchers sought and gained approval from the King Saud University's Institutional Review Board with Ref. No. KSU-HE-19558. The study did not invade the integrity of the participants. All the participants were issued verbal and written information concerning the objectives of the research and their specific roles. Participants were given anonymous names to safeguard their privacy and ensure that the results were not traced back to them. None of the respondents was pressured into participating in this study.

\section{Sampling and procedures of data collection}

Meetings were organized for potential participants at the selected wards in the hospital to explain the study's objective and what the participants would be asked to do. They were given information sheets containing the researcher's contact and additional information for further inquiry if required. They were permitted to make their own choices about their involvement. A purposive sampling method was used to recruit participants, following the inclusion criteria. According to developed criteria, only 
professional nurses and midwives employed as qualified nurses since these are the ones that majorly interact with patients, nurses with more than two years of experience, along with those with a diploma as the minimum qualification level, were regarded as the study population. Participants with insufficient experience with patient advocacy, empathy, and care were excluded from the research. The nurses worked in diverse settings hence created an opportunity to comprehend and compare their divergent perspectives.

A semi-structured guide was used to accumulate information from the participants. The interview guide was developed based on the purpose of the study and the literature gap. Open-ended questions were asked to allow participants to express their thoughts and to probe the participants' responses. The timing and place of the interviews were decided based on the convenience of the potential participants. Face-to-face interviews were conducted, and the participants were given anonymous names to safeguard their confidentiality. The interviews were performed in Arabic and audiotaped. The responses were transcribed, and an Arabic interpreter was consulted to translate the appropriate words into the English language. Data were collected from 25 February 2020 to 20 March 2020.

\section{Data analysis}

The principles of thematic analysis were applied to examine the data. Two expert researchers independently reviewed the transcripts to have them coded, categorized, and organized before creating a consensus upon the extracted meaning and significance of the analyzed data. The researchers relied on the MaxQDA software for coding and arrangement of the data. The themes of the research were extracted by organizing and structuring the transcribed data. Fragments of the interviews were compared to each other to derive the common factors.

\section{Trustworthiness}

To ensure the trustworthiness of this study, various strategies were adopted, including the use of a prior developed interview guide with one well-trained interviewer for conducting all interviews. A detailed description of the research background, its method, and sample background were provided to allow for findings' transferability in similar contexts. In-depth interviews facilitate full exploration of the nurses' traits that affect patient advocacy (Merriam \& Tisdell, 2015). Also, Kalu and Bwalya (2017) emphasized the benefits of documenting common themes, as it helps to identify recurring patterns and themes and ensure that participants' perspectives are fully understood during data analysis. In congruence with these recommendations, we transcribed the discussion, read the document carefully, and then identified and coded the emerging themes. In addition, the final analysis was presented back to the respondents to assess and make relevant comments. This method was used to maintain the collected data's credibility and trustworthiness of results by ensuring that the researchers had comprehended and correctly interpreted the study findings.

\section{RESULTS}

According to developed criteria of inclusion and exclusion for the current study, twentyone midwives and professional nurses were sampled purposively from a population of 
761 nurses. Fourteen of participants were female, and males were represented by seven participants. Nurses from Med/Surg wards were nine nurses; and five nurses were recruited from OB/GYN. Cardiology and Oncology wards were represented by four and three nurses, respectively.

The transcribed information was structured and organized in various categories and used to extract the themes. Furthermore, the respondents formulated these elements, which they considered significant in offering eminent nursing care to their patients. The elements/themes are expounded below.

\section{Empathy}

Initially, it was considered beneficial to obtain insight into the interviewees' acuities on empathy for developing a clear explanation of this study's findings. They were asked to provide their definitions of kindness. The answers were likewise common to many interviews:
"Well, in my opinion, empathy is the type of feelings that can be shared or appear to the other person as an expression of coherence with what they pleased or suffered. It is usually presented in the form of expressions of happiness, reassurance, or fondling." (P 01).
"It is how much someone understands others' situation and tries to feel what they are feeling and see things from others' perspective.' (P 04).
"An empathetic relation is friendly, close and cordial, which allows to understand patients from a closer view resulting in the increased possibility of understanding patient's perspective." (P 10).

Openness to the patient was considered as an essential aspect and viewed as an attitude and communication skill. To be precise, honesty is the individual capacity to have dignity for the feelings and thoughts of a patient:

"As a nurse, empathy can be conveyed through good communication and showing sincere curiosity. Requesting others to share their opinions, moods, insights, and practices is one of the most effective and unswerving ways to convey compassion and comprehension. We, as nurses must also spend some time with our patients and make them involved in each part of care and showing their right to refuse what is seen as contrary by them to their preferences or beliefs. I believe building a good therapeutic relationship with a patient is relying on the empathic skill of the nurse." (P 03).

"Someone can convey empathy to another through a positive attitude, positive listening, sharing joys, and sorrows. As a nurse, we can convey empathy by building a good relationship with the patient, meeting his or her needs, and respecting his or her values and beliefs. We can also help a patient to relax by spending time with him, listening and addressing his or her concerns, answering all of his questions, explaining each nursing procedure to the patient, and clarifying its benefits to his health. Similarly, I will ensure to defend the patient's discretion as a top priority." (P 06). 
"For me, my relationship with the patient is good when I try to be more communicative and interactive with the family, regarding patients improved developments, treatment preferences, and doubts. This further help me in removing all doubts from the patients' mind. It makes patients and their families more confident regarding the provided treatment." (P 12).

The insight of nurses was more inclined towards empathy as compared to sympathy. They explained sympathy as a more emotionally interacted procedure, where nurses make efforts to accustom to the patients' emotions by acknowledging their pain. Nurses experienced this as a gentle and warm endeavor for comprehending patients' emotional state:

"Of course, the nurse practicing empathy will act in harmony with the patient. In addition, they would build a good therapeutic relationship. This relationship is built on trust and respect and meeting the patient's needs. Therefore, it clearly affects patient satisfaction positively." (P 02).

"The nurse can convey empathy in different ways. There is no definite algorithm for showing empathy. Depending on the patient's situation, the nurse can show empathy by listening, committing to sit whenever the patient is in need to talk, expressing needs, emotions, or emotional status even in joyous moments, encouraging the patient, acknowledging their pain, and supporting whenever the need arises." (P 05).

The majority of the respondents consider non-verbal communication as an essential emphatic skill. They thought it was maintaining eye contact, leaning forward or backward to emphasize listening, and showcasing an interesting facial expression. Some participants identified physical contact's significance, such as offering a handkerchief, a hand on the shoulder, or an embrace when the patient is crying.

"Nursing care is not measured by words, but there are actions that reflect empathy. Nursing actions lead to the development of positive results on the client's health. The patient's satisfaction is affected positively and negatively by the nurse's reaction, If the nurse portrays great empathy and administers good nursing care that meets all the patient's psychological and physical needs within a professional framework, this may lead to high patient satisfaction. It should be noted that the empathy of the nurse is also affected by a combination of factors such as the work environment, the culture of the institution, and nurse's job satisfaction." (P 07).

Nurses also indicated that different verbal skills were used in patients' emphatic care, such as summarizing and reflecting on the feelings and thoughts of patients, clarifying the question, displaying pauses and relaxed tone of voice, and exciting listening. Attention to cues and reference to previous events or consults in the patient's life was the specifically mentioned skills.

"In certain cases, conflicts take place when I am unable to communicate them things regarding treatment and patient's condition effectively. This further effect 
my relationship with the patient, making him/her more doubtful regarding the diagnosis and treatment outcomes. For me, communication is the key to balance the relationship." (P15).

"Constant communication with patient is a part of providing quality care to patients. Poor communication takes place, when there lies a weak relationship between nurse and patient which further affect overall treatment outcomes." (P $14)$.

A critical and differentiating aspect of empathy was the closeness of the patient concerning the patient's suffering. Empathy needed the nurse to approach a patient's suffering in a susceptible approach compared to sympathy, which entailed patients' emotionally distancing themselves from suffering through an overly misguided or demonstrative reaction or avoidance. Nurses have also mentioned the positive effects of empathy. Most nurses were convinced that empathy positively contributes to a better explanation of complaints and enhanced diagnostics, therapy adherence, and receiving detailed and useful information. This act allowed nurses to deal efficiently with the patient's concerns and achieve successful treatment.

"Yes, of course, the goal of empathy is to make the quality of our patients better. So, by practicing this competence in our daily practice, we definitely will enhance patient satisfaction. Since we meet the needs of our patients according to their values and preferences and we also build a good relationship with them." (P 03).

"The nursing profession is based on empathy. To be a nurse you must be an empathic person. From my viewpoint, I found it is connected to the patient's gratification and health outcomes. If a nurse is more empathic, her patient will be more satisfied with the quality of care he receives and vice versa." (P 06).

\section{Advocacy}

Advocacy from the perspective of nurses includes being nurturing, assertive, persistent, and empathetic. The participants were convinced that they have the traits that enable them to advocate for the patients effectively as they are compassionate towards their suffering. They also spent additional time nurturing their patients, thus recognized their needs. They observed themselves as very accessible and approachable to patients, which facilitated them to confide in them. Besides, nurses helped patients and educated them on finding their ways via the health system. They usually became alternate mothers to infants, resolved misunderstandings, and comforted angry patients.

Nurses also expressed that they saw patients' advocacy as an ethical duty and encouraged patient engagement. They also respected the patients' rights and assured them confidentiality in their advocacy. Most of the nurses (20 out of 21) held that they were more conversant with the problems affecting the patients and were better positioned to advocate for them as they spend more time with patients while offering health care.

"We are in the department all the time with the patients. We interact with them, hand over patients, and write reports on them so we are aware of whatever is 
wrong with them. I think we [nurses] are the best to stand in for the patients." (P $11)$.

Nurses were also convinced that they were accessible and approachable to the patients rather than other health professionals to advocate for the patients effectively.

"Nurses are the leading people at the hospital and patients approach us whenever they need to talk. They feel shy approaching other health care officers and do not comfortably ask questions." (P 13).

It was also observed that nurses offered health education and counseled mothers as part of their nurturing trait.

"We educate them on the dos and don'ts, exercise, diet, and drugs." (P 17).

"Sometimes we had to convince mothers to get treatment despite the pressure of her family. We explain the pros and cons of being treated and the confidence is built through this compassion." (P 18).

One of the nurses has expressed that sometimes they [nurses] play mothers' roles and take care of the patient's children in their absence.

"We let the child stay in the department and the nurses would take care of the child. If we identify that there are other children at home and no one is available to take care of them, we can come and stay with the child in the department. This kindness will help the mother to go and return in the morning" (P 21).

Some of the male nurses have resolved misunderstandings and comforted anguished patients.

"Sometimes, we have to deal with the situation where patients have misunderstandings related to bills and we have to mediate and explain things to their satisfaction" (P 19).

"Usually, the patients are insulted by some staff members on the premises. We [nurses] resolve the dispute and calm the patients, and then talk to the staff to deal with the patients with professionalism" (P 16).

\section{Caring}

It was observed that the compassion and understanding of the patients' problems were the core aspects of nursing care. Sometimes, they had to become patients' voices and argue with the hospital management to fulfill patients' needs and consequently offered patients financial and material assistance. They also enabled their care, allowed them access to their caregivers whenever possible, and updated anxious relatives on the patient's condition. It was observed that most nurses realized and understood the feelings and challenges of patients and, therefore, communicated their needs and appealed on their behalf. 
"Patients are served meals in the morning and afternoon sessions. We [nurses] also propose management to serve meals to patients three times a day, and the meals should be served before they take their medications." (P 11).

The viewpoints of nurses indicated that quality care is the preferable approach to providing patient care (Gröndahl, Muurinen, Katajisto, Suhonen, \& Leino-Kilpi, 2019). This care is delivered safely based on appropriate nursing standards regardless of discriminatory behaviors throughout the caring process.

"We usually abide by the standards, whenever we are adopting a particular format. The rules cannot be omitted. I have made efforts to make them flexible for the provision of standard quality care." (P 13).

One of the participants expressed about the importance of excellent care for patient satisfaction, as follows:

"I provide the best care to my patients as I always put the satisfaction of my patient first. My behavior toward the patient will not be changed if my patient is illiterate or poor. The importance is towards offering excellent care." (P 09).

The adherence to safety was also regarded as another measurement of providing eminent care:

"You cannot omit medication of patients or mistakenly provide their medication twice. We have to remain conscious and precise in all of these duties and concerns." (P 20).

Nurses have expressed that they attempt to offer adequate care to eliminate patient pain and enhance their condition for attaining preferable caring outcomes. In this instance, a nurse revealed:

"I certainly do my best by assuring that my patients feel less pain so that my care is effective for them. I also help my patients who are about to pass away, so that they are comfortable and, in less pain." (P 08).

\section{DISCUSSION}

This study was conducted by interviewing twenty-one Saudi nurses working in different hospital settings at the King Saud Medical City in Riyadh, Saudi Arabia, to ascertain their perspectives on how empathy, advocacy, and caring can be used to measure quality nursing care. The respondents averred that nurses should incorporate these elements into daily nursing practice to increase positive patient feedback and quality nursing care. This study reveals that empathy, advocacy, and caring are significant components of quality nursing care.

\section{Empathy}

By evaluating the collected data, it became apparent that nurses frequently apply empathic skills during their regular day-to-day work. According to the respondents, 
verbal and non-verbal communication was vital in the provision of quality nursing care. Nurses should continuously interact with their patients to comprehend their thoughts, pain, and reactions to the medicine. However, nurses should avoid being emotionally attached to patients. Nurse-patient communication is essential for understanding the needs of patients and providing critical care for the patients. For durable interaction, empathy is necessary between patients and nurses (Usta, Demir, \& Yagmuroglu, 2012). Enhancement in empathic skills offers practical communication skills for nurses (Vaccaro et al., 2019). This study's findings indicated that there might be deleterious effects of being overly sensitive to others' pain and suffering, even though empathy is a fundamental aspect of nurse-patient interaction. This effect is specifically observed when nurses lack self-compassionate abilities. These findings are parallel to the finding proposed in the study of Duarte et al. (2016); according to the study, nurses who are empathetic must reflect increased self-compassion for a better quality of life. Nurses who are highly empathetic result in developing compassion fatigue and increased nurse burnout resulting in negatively affecting the quality of life of nurses. In addition, studies suggested that self-compassion in various healthcare professionals is beneficial for the clinician's well-being. It is further important to enhance the clinician's potential of showing compassionate care for patients (Boellinghaus, Jones \& Hutton, 2014; Raab, 2014; Mills \& Chapman, 2016).

Training might be significant for avoiding compassion fatigue as well as for promoting compassion satisfaction in self-compassion. This training can also encourage empathetic care, which has been indicated to significantly influence patients' health outcomes and professionals' well-being, considering the close interaction between empathy for patients and self-compassion. Previous studies have shown mindfulness-based interventions to be an influential approach to developing self-compassion and compassion for patients (Birnie, Speca, \& Carlson, 2010; Tirch, 2010; Wallmark, Safarzadeh, Daukantaite, \& Maddux, 2013). According to Moudatsou et al. (2020), nurses can create an effective therapeutic nursing care plan tailor-made for the patient's needs through an empathetic relationship with their patients, thus promoting and delivering quality health care. Previous studies have also indicated that nurses' burnout can be reduced by different interventions, such as mindfulness-based interventions. We found that empathy can be used as a factor in gauging the value of nursing care. Other nurses suggested communication as the key to developing an effective relationship with patients since an incomplete or miscommunication between participants may lead to poor satisfaction of patients with the treatment (Molina-Mula, \& Gallo-Estrada, 2020). Respondents further suggested that nurses should be more open towards their patients; this help in eliminating the doubts regarding the treatment from patients' mind.

\section{Caring}

The comprehension of quality nursing care explained in this study recalls previous literature identifying caring as a notion interactive to nursing and considered its essence. The meaning of quality nursing care comprises the contemporary practicing nurses specified in this study based on outcomes, focus, and consequences that add knowledge for the nursing discipline. Modern practicing nurses may drive improvements in nursing quality care by additionally defining the profession. The quality of care rendered to the patient is highly reliant on the nurse-patient relationship. A good nurse-patient 
relationship improves the nurses' quality of care, improves patient satisfaction, and reduces hospital stays (Molina-Mula \& Gallo-Estrada, 2020). Positive caring behaviors influence the patients' physical and psychological aspects positively (A'la, Setioputro, \& Kurniawan, 2018). According to World Health Organization (2019), care provided to the patients is of high quality when the following factors are considered, which includes delivering healthcare that is safe and involves minimum to zero risks for patients along with the least possibility of medical errors. Next, care provided to patients must be effective and should be based on scientific knowledge and proven guidelines. It must also be timely, that is without any delays in the overall process. Other characteristics include the efficiency of the involved human and technological resources, following similar patterns of patient care, without any gender, race, ethnic, and geographical considerations (World Health Organization, 2020).

The application of caring nurse conducts includes the provision of safety and positive communication, as well as motivation to patients to adhere to care advice and treatment plans (Thomas, Newcomb, \& Fusco, 2019; Hajinezhad \& Azodi, 2014). In this study, the participants provided that patient satisfaction is of utmost importance hence the need to provide quality care to them.

\section{Advocacy}

Nursing advocacy is paramount in the delivery of excellent health care in health organizations. Most of the participants stated that the best way of promoting advocacy was through patient education on medical conditions, prescribed medications, and nursing interventions. They aver that they also offer maternal care and moral support to the family of the patients and arbitrating patient disputes. Kolawole and Adejumo (2020) avow that vulnerable patients are empowered and delivered from inept superfluous treatment through nursing advocacy. Nevertheless, several factors hinder nurses from exercising this significant role. They include a lack of nurse autonomy, poor relations between hospital management, caregivers, nurses, uncooperative patients, and ineffective communication between nurses and patients (Nsiah, Siakwa, \& Ninnoni, 2020). Additionally, poor patient advocacy can cause unavoidable health complications and death. Abbasinia et al. (2020) identified different attributes of advocacy, which include providing information to patients regarding their diagnosis, preferred treatment, involved complexities, and expected treatment outcomes. It further includes respecting patient's privacy, providing the choice to patients regarding the preferred treatment, and acting on a patient's belief.

Previous studies concur with the findings of this research that empathy, advocacy, and care are essential elements of improving patient experience. However, none of them explicated how these fundamental factors can be used to measure the quality of nursing care. This new and vital knowledge can be used by practicing nurses, instructors, legislators, and scholars. These findings offer an introductory experience that the essence of superior nursing care is allocated to nurses. Besides, this shared information is reflected from the nursing art that might be surprising for practicing nurses as it contradicts the fundamental emphasis. Eminent nursing care skills were focused on these nursing engagements with patients and were identifiable in nursing practices and peers. The three vital themes of caring, compassion, and advocacy were authenticated as 
the meaning of excellent nursing in practicing nurses' experiences. Nursing care is defined as to professionally abide on the healthcare standards while prioritizing patient's satisfaction. Considering and valuing the patient's safety is another important characteristic of increased patient care.

Through the above findings, empathy can be defined by the following characteristics of nurses, which include; sharing or feeling the emotions of other people, being more open in communicating patients' feelings, mood, suffering, preferences, or beliefs, to develop a relationship with patients which is entirely based on trust and emotions, and using and implementing appropriate verbal skills to develop clarity in patients regarding the provided treatment. Advocacy, on the other hand, can be defined as an ethical duty towards patients, which is fulfilled by being more approachable and accessible towards patients, providing them the required information and knowledge via counseling, while showing kindness through increased care. These common meanings can be applied to enlighten the nursing practice and appraise the value of nursing care being offered. Therefore, nurse administrators can create plans for aiding nurses effectively in the realization and delivery of excellent nursing care contemplative to the meaning of caring, compassion, and advocacy. They can apply these themes to assess the value of nursing care by identifying nurses' communication and behaviors with patients. Most notably, the majority of the nurses have expressed quality nursing care comparative to end-of-life situations.

Additional studies are required to comprehend the essence of this finding and to further extend the information by collecting the information through quantitative analysis for more explicit dimensions of care, empathy, and advocacy, which act as a limitation of the present study.

\section{CONCLUSION}

In conclusion, patient advocacy, empathy, and caring are part and parcel of a nurse's characteristics. They are fundamental attributes in the assessment of the value of nursing care given to patients. Empathy and advocacy are critical skills that nurses should portray when caring for patients. They aid in listening to people and comprehending their views, particularly in the nursing practice. Empathy skills are essential while providing care to patients. Nurses caring for patients must be assessed for both education and empathy skills during employment. Even though education is a direct relationship between attitudes and empathy skills, it cannot explain patients' care.

Additional studies can be carried out to evaluate the relationship between attitudes and empathy skills towards patients. The healthcare system must stimulate nurses to think and act beyond their usual roles and engage in continuous learning to maximize healthcare system consequences concerning patient-perceived care quality and care outcomes. The findings and suggestions of this study might be utilized by senior nursing managers in programs that are devised for enhancing the quality of nursing care. 


\section{ACKNOWLEDGMENT}

The authors are very thankful to all the associated personnel in any reference that contributed to/for this research.

\section{CONFLICT OF INTEREST}

The author affirms no opposing interest.

\section{REFERENCES}

Abbasinia, M., Ahmadi, F., \& Kazemnejad, A. (2020). Patient advocacy in nursing: A concept analysis. Nursing Ethics, 27(1), 141-151. doi:10.1177/0969733019832950

A'la, M. Z., Setioputro, B., \& Kurniawan, D. E. (2018). Nursing students' attitudes towards caring for dying patients. Nurse Media Journal of Nursing, 8(1), 25-34. doi:10.14710/nmjn.v8i1.17270

Aupia, A., Lee, T., Liu, C., Wu, S. V., \& Mills, M. E. (2018). Caring behavior perceived by nurses, patients and nursing students in Indonesia. Journal of Professional Nursing, 34(4), 314-319. doi:10.1016/j.profnurs.2017.11.013

Austin, W. (2000). Nursing, empathy and perception of the moral. Journal of Advanced Nursing, 32(1), 235-242. doi:10.1046/j.1365-2648.2000.01440.x.

Birnie, K., Speca, M., \& Carlson, L. E. (2010). Exploring self-compassion and empathy in the context of mindfulness-based stress reduction (MBSR). Stress and Health, 26(5), 359-371. doi:10.1002/smi.1305

Boellinghaus, I., Jones, F. W., \& Hutton, J. (2014). The role of mindfulness and lovingkindness meditation in cultivating self-compassion and other-focused concern in health care professionals. Mindfulness, 5(2), 129-138. doi:10.1007/s12671-0120158-6

Dadzie, G., Aziato, L., \& Aikins, A. D. G. (2017). We are the best to stand in for patients: a qualitative study on nurses' advocacy characteristics in Ghana. BMC Nursing, 16(1), 1-8. doi:10.1186/s12912-017-0259-6

Davoodvand, S., Abbaszadeh, A., \& Ahmadi, F. (2016). Patient advocacy from the clinical nurses' viewpoint: A qualitative study. Journal of Medical Ethics and History of Medicine, 9, 1-8. doi:10.1177/0969733016629772

Duarte, J., Pinto-Gouveia, J., \& Cruz, B. (2016). Relationships between nurses' empathy, self-compassion and dimensions of professional quality of life: A crosssectional study. International Journal of Nursing Studies, 60, 1-11. doi:10.1016/j.ijnurstu.2016.02.015

Elewa, A. H., ElAlim, E. A., \& Etway, E. G. (2016). Nursing interns' perception regarding patients' rights and patients' advocacy. SOJ Nursing \& Health Care, 2(3), 1-6. doi:10.15226/2471-6529/2/3/00123

Gröndahl, W., Muurinen, H., Katajisto, J., Suhonen, R., \& Leino-Kilpi, H. (2019). Perceived quality of nursing care and patient education: A cross-sectional study of hospitalised surgical patients in Finland. BMJ Open, 9(4), e023108. doi:10.1136/bmjopen-2018-023108

Hajinezhad, M. E. \& Azodi, P. (2014). Nurse caring behaviors from patients' and nurses' perspective: A comparative study. European Online Journal of Natural and Social Sciences, 3(4),1010-1017. Retrieved from http://europeanscience.com/eojnss/article/download/1300/pdf 
Hannan, J., Sanchez, G., Musser, E. D., Ward-Peterson, M., Azutillo, E., Goldin, D., ..., \& Foster, A. (2019). Role of empathy in the perception of medical errors in patient encounters: A preliminary study. BMC Research Notes, 12(1), 1-5. doi:10.1186/s13104-019-4365-2.

Heidke, P., Howie, V., \& Ferdous, T. (2018). Use of healthcare consumer voices to increase empathy in nursing students. Nurse Education in Practice, 29, 30-34. doi:10.1016/j.nepr.2017.11.007

James, R. B., \& Mill, J. E. (2018). Developing confidence for patient advocacy: Reflections from an international clinical experience. International Journal of Nursing Student Scholarship, 5, 1-8. Retrieved from https://journalhosting.ucalgary.ca/index.php/ijnss/article/view/56841/42949

Kalu, F. A., \& Bwalya, J. C. (2017). What makes qualitative research good research? An exploratory analysis of critical elements. International Journal of Social Science Research, 5(2), 43-56. doi:10.5296/ijssr.v5i2.10711

Karaca, A., \& Durna, Z. (2019). Patient satisfaction with the quality of nursing care. Nursing Open, 6(2), 535-545. doi:10.1002/nop2.237

Kim, J. (2018). Factors influencing nursing students' empathy. Korean Journal of Medical Education, 30(3), 229- 236. doi:10.3946/kjme.2018.97

Kolawole, I. O., \& Adejumo, P. O. (2020). Nurses' view on impact of patient advocacy in oncology care at university college hospital, Nigeria. ARC Journal of Nursing and Healthcare, 6(1), 1-9. doi:10.20431/2455-4324.0601001

Kuipers, S. J., Cramm, J. M., \& Nieboer, A. P. (2019). The importance of patientcentered care and co-creation of care for satisfaction with care and physical and social well-being of patients with multi-morbidity in the primary care setting. $B M C$ Health Services Research, 19(1), 13. doi:10.1186/s12913-018-3818-y.

Merriam, S. B., \& Tisdell, E. J. (2015). Qualitative research: A guide to design and implementation. New York: John Wiley \& Sons.

Mills, J., \& Chapman, M. (2016). Compassion and self-compassion in medicine: Selfcare for the caregiver. The Australasian Medical Journal, 9(5), 87-91. doi:10.4066/AMJ.2016.2583

Molina-Mula, J. \& Gallo-Estrada, J. (2020). Impact of nurse-patient relationship on quality of care and patient autonomy in decision-making. International Journal of Environmental Research and Public Health, 17(3), 835-847. doi:10.3390/ijerph17030835

Molina-Mula, J., \& Gallo-Estrada, J. (2020). Impact of nurse-patient relationship on quality of care and patient autonomy in decision-making. International Journal of Environmental Research and Public Health, 17(3), 835. doi:10.3390/ijerph17030835

Moudatsou, M., Stavropoulou, A., Philalithis, A. \& Koukouli, S. (2020). The role of empathy in health and social care professionals. Healthcare, 8(1), 26. doi:10.3390/healthcare8010026

Nsiah, C., Siakwa, M., \& Ninnoni, J. P. (2019). Registered nurses' description of patient advocacy in the clinical setting. Nursing Open, 6(3), 1124-1132. doi:10.1002/nop2.307

Nsiah, C., Siakwa, M., \& Ninnoni, J. P. (2020). Barriers to practicing patient advocacy in healthcare setting. Nursing Open, 7(2), 650-659. doi:10.1002/nop2.436 
Raab, K. (2014). Mindfulness, self-compassion, and empathy among health care professionals: a review of the literature. Journal of Health Care Chaplaincy, 20(3), 95-108. doi:10.1080/08854726.2014.913876.

Ratka, A. (2018). Empathy and the development of affective skills. American Journal of Pharmaceutical Education, 82(10). doi:10.5688/ajpe7192

Salimi, S., \& Azimpour, A. (2013). Determinants of nurses' caring behaviors (DNCB): Preliminary validation of a scale. Journal of Caring Sciences, 2(4), 269-278. doi: $10.5681 /$ jcs.2013.032

Thomas, D., Newcomb, P., \& Fusco, P. (2019). Perception of caring among patients and nurses. Journal of Patient Experience, 6(3), 194-200. doi:10.1177/2374373518795713

Tirch, D. D. (2010). Mindfulness as a context for the cultivation of compassion. International Journal of Cognitive Therapy, 3(2), 113-123. doi:10.1521/ijct.2010.3.2.113

Usta, Y. Y., Demir, Y., \& Yagmuroglu, H. (2012). Nurses' perspective on positive attitudes to cancer patients in Turkey: a qualitative study. Asian Pacific Journal of Cancer Prevention, 13(4), 1225-1229. doi:10.7314/apjcp.2012.13.4.1225

Vaccaro, L., Shaw, J., Sethi, S., Kirsten, L., Beatty, L., Mitchell, G., ..., \& Turner, J. (2019). Barriers and facilitators to community-based psycho-oncology services: A qualitative study of health professionals' attitudes to the feasibility and acceptability of a shared care model. Psycho-oncology, 28(9), 1862-1870. doi:10.1002/pon.5165

Wallmark, E., Safarzadeh, K., Daukantaite, D., \& Maddux, R. E. (2013). Promoting altruism through meditation: An 8-week randomized controlled pilot study. Mindfulness, 4(3), 223-234. doi:10.1007/s12671-012-0115-4

World Health Organization. (2020). Maternal, newborn, child and adolescent health, and ageing. Retrieved from https://www.who.int/teams/maternal-newborn-childadolescent-health-and-ageing/quality-of-care 